$\underline{\text { Supporting Information }}$

\title{
Synthesis and Microphase Separation of Poly(styrene- $b$-acrylonitrile) Prepared by Sequential Anionic and ATRP Techniques
}

\author{
Julie M. Leiston-Belanger, Jacques Penelle, Thomas P. Russell*
}

Table A. PS- $b$-PAN samples.

The data from this table was used to create Figure 3 in the manuscript.

\begin{tabular}{|c|c|c|c|c|c|c|c|}
\hline Polymer & $\begin{array}{l}\text { Reaction Time } \\
\text { (min) }\end{array}$ & $\begin{array}{c}\text { Total } \\
\mathrm{Mn}\end{array}$ & $\begin{array}{l}\mathrm{M}_{\mathrm{n}} \mathrm{PAN} \\
{(\mathrm{NMR})^{a}}^{2}\end{array}$ & Conversion $^{b}$ & $\begin{array}{l}\text { Volume }^{c} 7 \\
\% \text { PAN }\end{array}$ & $\begin{array}{l}\text { Total } \mathrm{M}_{\mathrm{n}} \text { of diblock } \\
(\mathrm{GPC})^{d}\end{array}$ & $\mathrm{PDI}^{\mathrm{e}}$ \\
\hline SANa1 & 2 & 2510 & 510 & 1.4 & 18.7 & 11100 & $\sim 1.11$ \\
\hline SANb1 & 10 & 3300 & 1300 & 20.5 & 37.1 & 18000 & 1.19 \\
\hline SANb2 & 20 & 3800 & 1800 & 27.4 & 44.9 & 20000 & 1.28 \\
\hline SANc1 & 30 & 4050 & 2050 & 38.7 & 48.1 & 21300 & 1.34 \\
\hline SANb3 & 30 & 4200 & 2200 & 30.5 & 49.9 & 22600 & 1.30 \\
\hline SANb4 & 40 & 4400 & 2400 & 41.6 & 52.1 & 22500 & 1.36 \\
\hline SANb5 & 50 & 4650 & 2650 & 36.4 & 54.6 & 24800 & 1.34 \\
\hline SANb6 & 60 & 5300 & 3300 & $N / A^{\dagger}$ & 59.9 & 27700 & 1.26 \\
\hline SANa2 & 60 & 4610 & 2610 & 37.9 & 54.1 & $\mathrm{~N} / \mathrm{A}^{f}$ & $\mathrm{~N} / \mathrm{A}^{f}$ \\
\hline SANc2 & 60 & 4790 & 2790 & 45.9 & 55.8 & 25700 & 1.385 \\
\hline SANc3 & 120 & 5260 & 3260 & 53.1 & 59.6 & 26000 & 1.48 \\
\hline SANc4 & 150 & 5300 & 3300 & 56.3 & 59.9 & $\mathrm{~N} / \mathrm{A}^{f}$ & $\mathrm{~N} / \mathrm{A}^{f}$ \\
\hline SANa3 & 180 & 5670 & 3670 & 54.1 & 62.4 & $\mathrm{~N} / \mathrm{A}^{f}$ & $\mathrm{~N} / \mathrm{A}^{f}$ \\
\hline SANc5 & 180 & 5650 & 3650 & 58.5 & 62.3 & 25900 & 1.57 \\
\hline SANc6 & 240 & 6000 & 4000 & 62.0 & 64.4 & 32600 & 1.46 \\
\hline
\end{tabular}

$\boldsymbol{a} \mathrm{M}_{\mathrm{n}}$ was determined using the dried polymer sample in DMSO- $\mathrm{d}_{6} \boldsymbol{b}$ Conversion was measured using aliquots of the reactions $(\mathrm{a}, \mathrm{b}, \mathrm{c})$ at different times and using the integration of the DMF peak at $7.95 \mathrm{ppm}$ as an internal standard to measure the disappearance of the monomer peaks. $\boldsymbol{c}$ Volume fraction was calculated from the NMR data using $d_{P S}=1.06 \mathrm{~g} / \mathrm{ml}$ and $d_{\text {PAN }}=1.17 \mathrm{~g} / \mathrm{mol}$. $\boldsymbol{d}$ Significantly higher $\mathrm{M}_{\mathrm{n}}$ using SEC is typical of PAN containing samples when compared to NMR; SEC relative to PS standards. $\boldsymbol{e}$ No correction was done to account for peak tailing from instrument, which may account for a broadening in PDI (see Figure 3). $f$ Data is not available due to limited quantities of material. 


\section{SEC results for PAN polymers}

The molecular weight characterization of PAN containing homopolymers and diblock copolymers can be tricky. Characterization of PAN using NMR is limited to samples that have known internal standards (such as a well-characterized first block in the case of a diblock copolymer) or are of low enough molecular weight for end-group analysis. The next typical step for molecular weight characterization, especially when an analysis of the molecular weight distribution is needed, is to use SEC, usually in DMF. PAN-containing polymers interact with typical SEC column packing materials resulting in a broadening of the distribution by tailing, or odd peak shapes, that can be strongly influenced or screened by the addition of DMF soluble salts. ${ }^{1,2}$ Even with the addition of salts to screen interactions, the peak shape can still be influenced by impurities such as water, the type or brand of SEC packing material, and even the age of the columns (as per discussions with Polymer Lab technicians).

Also, when calibration standards such as PMMA or PS are used, the molecular weights corresponding to the PAN peaks can be off by an order of magnitude, which has been seen here (see Table A and Figure B), and in other diblock copolymer studies where PAN is one of the blocks ${ }^{3,4}$. Since, for our study, we used a known first block of PS-Br and were able to use that for the determination of the Mn of the second block, there was no need to use the SEC data to determine molecular weight. The data is included below for completeness, and for those that may encounter similar difficulties in PAN characterization. 
Figure A: Molecular Weight distribution by SEC using DMF (0.01 M LiCl), 0.5 $\mathrm{mL} / \mathrm{min}$. Peak tailing is evident in all samples, and has not been corrected for in calculations, (see table A).

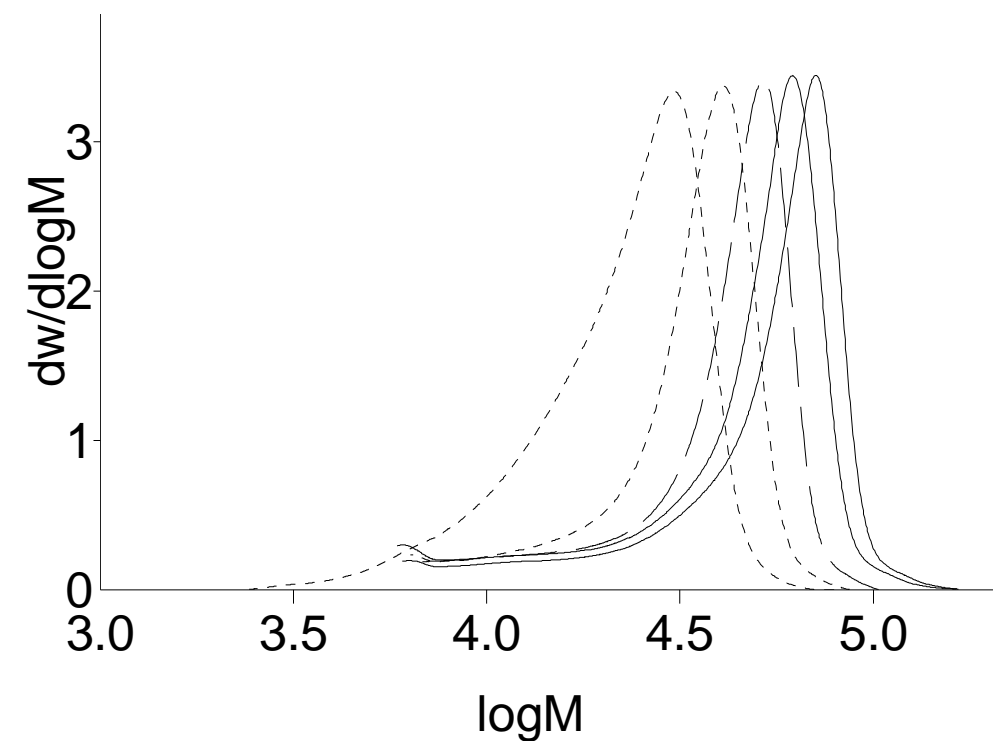

Figure B: Difference in the number average molecular weight between NMR and GPC measurements.

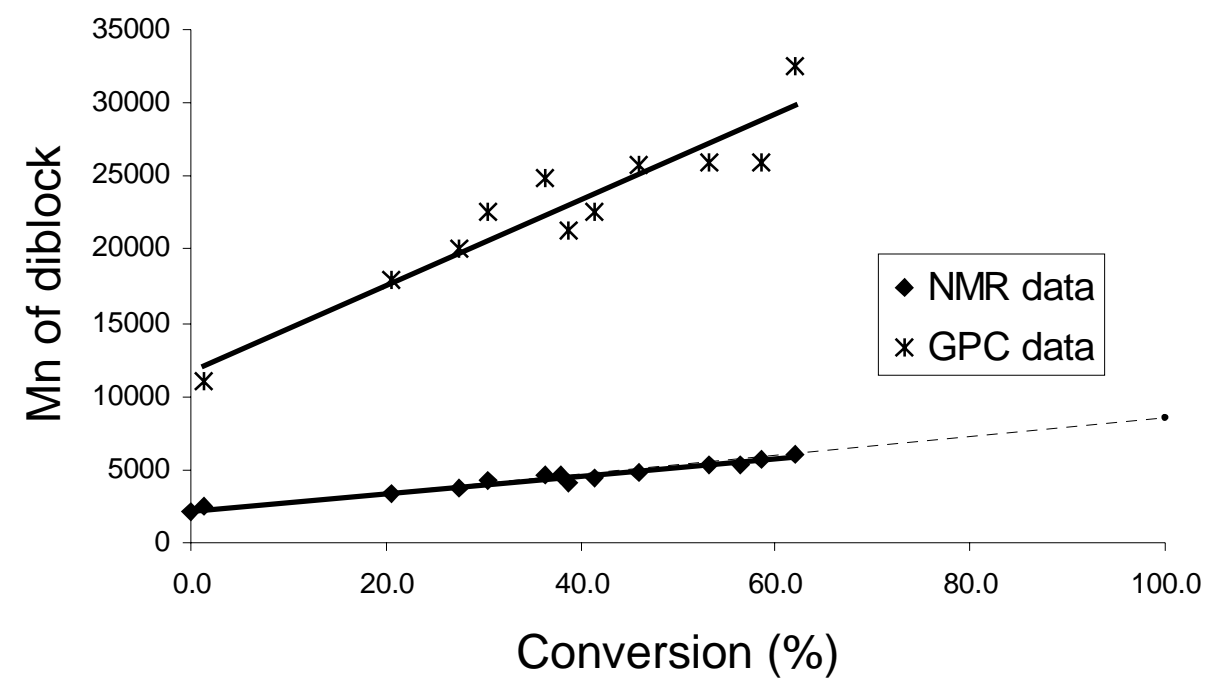

\section{References}

(1) Azuma, C.; Dias, M. L.; Mano, E. B. Polym. Bull. 1995, 34, 585-592.

(2) Azuma, C.; Dias, M. L.; Mano, E. B. Polymer Bulletin (Berlin) 1995, 34, 593598.

(3) Tang, C.; Kowalewski, T.; Matyjaszewski, K. Macromolecules 2003, 36, 14651473.

(4) Kowalewski, T.; Tsarevsky, N. V.; Matyjaszewski, K. J. Am. Chem. Soc. 2002, $124,10632-10633$. 